\title{
Characterization of Phages Virulent for Sarothamnus scoparius Bradyrhizobia
}

Wanda Małek, ${ }^{1}$ Ewa Sajnaga, ${ }^{1}$ Sylwia Wdowiak-Wróbel, ${ }^{1}$ Bożena Studzińska, ${ }^{1}$ Izabela Święcicka, ${ }^{2}$ Izabela Nosalewicz, ${ }^{1}$ Marta Słomka, ${ }^{1}$ Agnieszka Tatara, ${ }^{1}$ Antoni Gawron ${ }^{3}$

${ }^{1}$ Department of General Microbiology, M. Curie-Skłodowska University, 19 Akademicka Street, 20-033, Lublin, Poland

${ }^{2}$ Department of Microbiology, University of Białystok, 20B Świerkowa Street, 15-950, Białystok, Poland

${ }^{3}$ Department of Comparative Anatomy and Anthropology, M. Curie-Skłodowska University, 19 Akademicka Street, 20-033, Lublin, Poland

Re: Curr Microbiol 51(4) 244-249: Due to an unfortunate error, the name of one of the above authors was missspelled in the online and print versions of this article (DOI: 10.1007/s00284-005-4557-y). It should read Izabela Święcicka. 